\title{
A Literature Review on Cognitive Accessibility
}

\author{
Terhi KÄRPÄNEN ${ }^{\mathrm{a}, 1}$ \\ ${ }^{a}$ Laurea University of Applied Sciences, Finland, Estonian Business School, Estonia
}

\begin{abstract}
The purpose of this study was to review the qualitative literature on cognitive accessibility in a digital environment and areas of inquiry for future qualitative research in this context. The focus of this literature review was to identify qualitative research in the cognitive accessibility field and how commonly this term is mentioned in qualitative research articles. In this study, a literature review was conducted on selected qualitative research studies performed globally related to cognitive accessibility. This literature review analysed through meta-synthesis. Based on the results of the literature review, an understanding of existing qualitative research was obtained in the cognitive accessibility field, as well as topics for further qualitative research in the cognitive accessibility field.
\end{abstract}

Keywords. Cognitive Accessibility, Digital Services, Universal Design

\section{Introduction}

As digitalization rapidly changes services to a digital environment in both the public and private sectors, many questions related to Universal Design arise. How to make digital services as cognitively accessible for people who have disabilities and thus provide a better digital service user experience for all users? Universal Design encompasses removing obstacles for people with disabilities, which entails retrofitting buildings as well as changing the methodology for designing new ones- 'barrier-free' design. Some of the Universal Design principles are related to accessibility in a digital environment. Universal Design can be defined as the design of products and environments that can be experienced by people of all ages and abilities [1-2].

There is growing interest in studying this field in order to understand the importance of cognitive accessibility when using digital services. Qualitative research provides a better understanding of digital service usage and possible accessibility barriers. Digital public services are routinely produced by national, state and local governments. The services are delivered to citizens, businesses and other entities under their jurisdiction. Public services should be universal and sensitive to the context where such services are delivered [3].

\footnotetext{
${ }^{1}$ Corresponding author: Terhi Kärpänen, Laurea University of Applied Sciences, EBS.
} 
Cognitive accessibility is part of the overall accessibility framework. Cognitive accessibility means that digital services are simple, consistent, clear, multi-modal, errortolerant and attention-focusing to use, taking into account all users. Cognitive accessibility benefits all users, but especially users who have cognitive and learning disabilities. There is a huge number of cognitive disabilities and variations of them. This population is larger than all other physical and sensory disabilities combined [4]. W3C [4] and The Finnish Association on Intellectual and Developmental Disabilities (FAIDD) [5] has stated that more research needs to be conducted in the cognitive accessibility field.

In providing technology that supports cognitive accessibility elements, people can choose how to interact with the content and the process or how to access information, e.g., navigating through content with different strategies, accessing information in many ways and personalizing the content. Digital service developers can avoid the barriers by taking into account accessibility requirements, thus optimizing the user experience. Cognitive accessibility means flexible content, users should have enough time to read and use the content, easy-to-navigate support, text and language should be understandable, web pages should operate in a predictable way and users should be able to correct and avoid mistakes $[4,6]$.

The European Union has set up Accessibility Requirements for Public Procurement of ICT Products and Services in Europe. The web and digital content requirements need to follow defined success criteria from the W3CWeb Content Accessibility Guidelines [7]. Unfortunately, some of the cognitive accessibility elements are not defined in the success criteria, such as proper language or content--related elements. This field needs more understanding and studies.

Making web accessibility improvements based on success criteria and adding cognitive accessibility elements will improve information access for everyone, not only for people with disabilities. Everybody benefits when digital services are easy-to-use and language is understandable. It may be argued that accessibility is primarily a moral idea, which as a definition is not without problems, since in speaking about accessibility, one always invokes barriers before subsequently pondering how to deconstruct them [8].

The general features of digital service usage, user experience and Universal Design principles for designing digital services are well-known. There are different qualitative research studies being conducted based on the cognitive accessibility of websites used earlier, especially faced by senior users of websites [9], and web practitioners' thoughts about web accessibility [10]. But more research conducted recently is needed in terms of combining digital service usage from the perspective of cognitive accessibility among people with different disabilities. This literature review looked for the research gaps in cognitive accessibility in a digital environment among qualitative research papers and articles. Qualitative synthesis is recognized as a valuable tool for examining participant's meanings, experiences and perspectives [11].

Despite much empirical research based on accessibility, much of it has focused on the disability itself or assistive technology, not cognitive accessibility in general. Most of the studies are focused on literature reviews, e.g. the studies by Borg et al. and Manzoor et al. [12-13], quantitative studies in the accessibility field, e.g. Sintov et al.'s [14] study or mixed-method studies, e.g. by Johansson et al. [15]. There is an insufficient amount of qualitative research. Qualitative studies bring more understanding of how people are using digital services or access to information and how good design and cognitive accessibility elements make the process easier for all users. Qualitative research aims to help us to understand the world in which we live and why things are the way they are, and it is concerned with finding answers to questions that begin with - 
Why..., How..., In what way... [16]. Qualitative studies can give all participants the possibility to participate, including people with disabilities, and they provide a more indepth understanding of the phenomena.

The aim of this study was to review the qualitative literature on cognitive accessibility in a digital environment and areas of inquiry for future qualitative research in this context. Understanding the enablers and barriers when using digital services, the literature review enables a more holistic understanding of the cognitive accessibility field through qualitative studies and its importance for the digital service design process and possible research gaps in qualitative research. Another area of interest was to understand how cognitive accessibility was generally mentioned in qualitative research articles.

\section{Methods - Conducting a metasynthesis}

A narrative literature review was conducted on selected qualitative research studies performed in Finland and other countries related to cognitive accessibility and its relation to digital service and public digital service usage in a digital environment. The purpose of the literature review was to reveal the problems and weaknesses in a particular area of investigation [17].

\subsection{Inclusion criteria}

The digital environment is changing rapidly, and thus one inclusion criterion was to get information and articles written in the last 5 years. This literature review focused not only on people with disabilities and cognitive disabilities but on all people who use digital services. The literature review consists of an overall understanding of the keyword 'cognitive accessibility', qualitative research and studies related to the cognitive accessibility field. The review sought to identify all qualitative studies related to cognitive accessibility in a digital environment. Thus, I excluded studies with quantitative methods and mixed methods, literature reviews and heuristic or other framework analysis and concentrated on qualitative studies such as interviews, observation, co-design, usability and prototype testing. The quantitative studies and studies using mixed methods were excluded because I wanted to focus on qualitative studies in this field. I also excluded papers that focused on other assistive technology use such as screen readers, although those are part of cognitive accessibility. The reason for that is its technical implementation, which was not the focus of this study. All articles in the search scope, excluding the ACM search, were peer-reviewed, purely qualitative research studies in English.

\subsection{Search strategy}

I conducted the keyword searches by using the keywords cognitive accessibility and digital services by using "OR and "AND" relations. I wanted to investigate how commonly cognitive accessibility has been used in a digital context at the same time, so the "Cognitive Accessibility" search word was mandatory at the beginning of the search process. In ACM and Scholar searches, I used only the "Cognitive Accessibility" term. The searches included cognitive accessibility, accessibility, digital service, digital public service, e-services, online and qualitative research. I searched for publications in various 
databases such as ESBCO, ACM, ProQuest and Google Scholar with the help of a professional librarian. ACM (Association for Computing Machinery) was selected due to the digital aspect of this literature review. In the ACM search, I was not able to identify whether the articles were peer-reviewed. After removing duplicates from all searches, I identified 110 articles in total that were related to accessibility, cognitive accessibility and digital services. The next step was to identify the articles that met the other criteria, e.g. purely qualitative studies and digital services without assistive technology. I identified the articles by screening the titles, keywords and abstracts with a combination of search words. Then I excluded the literature reviews, quantitative research, mixed method research, validation or guidelines, assistive technology -related articles and other articles that did not meet the criteria. The result was 7 articles, which I included in the literature review. A flow diagram shows the search strategy in Figure 1.

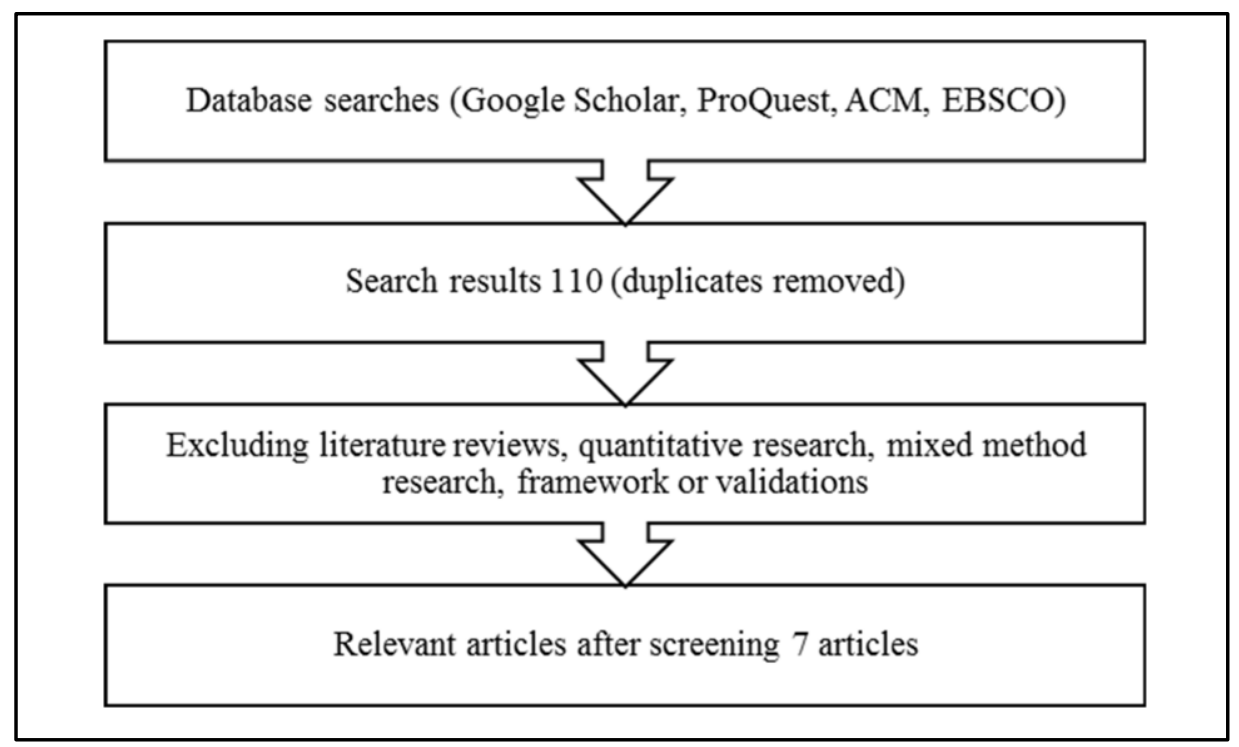

Figure 1. Search strategy flow diagram

\subsection{Data analysis}

The literature review focused on search qualitative researches among articles. The literature was analysed through qualitative metasynthesis. A metasynthesis is an interpretive process that integrates the results from different qualitative studies. The author of the synthesis seeks to make an interpretation based on the original interpretations, i.e. the research results of other researchers [18]. Metasynthesis is an important technique for qualitative researchers. The aim of metasynthesis in qualitative research is interpretive, and qualitative metasynthesis seeks to understand and explain phenomena rather than increase certainty in the causation. A metasynthesis attempts to integrate the results from a number of different but interrelated qualitative studies. 
The technique has an interpretive, rather than aggregating, intent, in contrast to a meta-analysis of quantitative studies [19]. The data analysis process started by reading the titles and abstracts of each article. Then the findings were coded and grouped by categories and finally in themes. The results of the metasynthesis consist of the themes that we developed in the analysis.

\section{Results}

This chapter presents the results by themes and the characteristics of the research. Most of the methods in the papers were quantitative methods or a mixed-method approach, so these papers were excluded. The majority of the reviewed articles were literature reviews or guidelines. Most of the articles were published during 2019 and 2020. One study was from the year 2015. The common methods in the articles were semi-structured interviews and observations. The most common analysis was thematic analysis. The sample sizes varied from 7 to 197 participants. The characteristics of the research are summarized in Table 1.

Table 1

Characteristics of the research

\begin{tabular}{|c|c|c|c|}
\hline Authors & Methods & Participa & \\
\hline & Data collection and analysis & $\begin{array}{l}\text { Sample } \\
\text { size }\end{array}$ & $\begin{array}{l}\text { Geographical } \\
\text { area }\end{array}$ \\
\hline Johansson et al. [20] & $\begin{array}{l}\text { Participatory action research, study circles } \\
\text { Thematic }\end{array}$ & over 100 & Sweden \\
\hline Cobigo et al. [21] & $\begin{array}{l}\text { Semi-structured interviews } \\
\text { Thematic }\end{array}$ & 10 & Canada \\
\hline Singleton et al. [22] & $\begin{array}{l}\text { Video observations and semi-structured } \\
\text { interviews } \\
\text { Comparative analysis }\end{array}$ & 7 & USA \\
\hline Watfern et al. [23] & $\begin{array}{l}\text { Semi-structured interviews and focus groups, } \\
\text { partly observation } \\
\text { Thematic analysis }\end{array}$ & 36 & Australia \\
\hline Koushik et al. [24] & $\begin{array}{l}\text { Interviews and observations } \\
\text { Open coding }\end{array}$ & 10 & USA \\
\hline González et al. [25] & $\begin{array}{l}\text { Co-design process, several methods } \\
\text { Analysis unspecified }\end{array}$ & 10 & Colombia \\
\hline Crabb et al. [26] & $\begin{array}{l}\text { Workshop, several methods } \\
\text { Interpreting research data, card sorting }\end{array}$ & 197 & UK \\
\hline
\end{tabular}


Overall, the studies referred to finding barriers in the accessibility and cognitive accessibility fields when using digital services [20-26]. The study participants varied in each study. In most of the studies, the participants were people with some kind of disability and identified as social groups. The study participants were mentally disabled persons [20], cognitive disabilities persons [24], technology developers and students [21, 26], visually impaired persons [23] and intellectually disabled persons [22, 25]. The studies' subjects varied. There were studies related to website barriers and development $[20,22]$, accessible documents [23] and overall accessibility [24-26] in all digital service contexts. One study focused more on digital service privacy [21].

The majority of studies that I examined were related to societal challenges to use digital services. The cognitive accessibility barriers are the same whether the field is in the public or private sector. Part of the challenge is a different group of people with disabilities, but the main statements were that the barriers are relevant to all people and not just a group of people with disabilities. The analysis of articles reveals different themes. The themes were: (1) data privacy, access to information and consent, (2) content and language and (3) designing digital services.

\subsection{Data privacy, access to information and consent}

Access to information seemed to be problematic especially for groups of people who have some disabilities. One relevant problem was found when trying to access information before using any digital services. The important observation was that some people with disabilities can have old computers or no access to a computer at home, and thus it is difficult to access public service information. The option is to use a library's computers, but there are usually time limitations for use. Some participants argue that many probably would change their opinion if given the opportunity to learn more and to test the devices and services [20].

Data privacy, log-in details and the consent process were difficult to understand. According to the participants, there is no good way to 'prove who you are' on the Internet [20]. Overall, participants met severe problems with login procedures, and some of the participants avoid solutions that require logging in. The participants feel that access to information is difficult to manage and use-especially log-in functions, setting up a password and username and the CAPTCHA process (an acronym for Completely Automated Public Turing test to tell Computers and Humans Apart). [20, 22]. These are a challenge for many participants who have disabilities, especially people with intellectual disabilities. The challenge was also to remember the password and username [22]. The log-in process should be planned very carefully when designing websites. Participants were not comfortable giving this information. A participant in Watfern et al.'s [22] study emphasized the problem: 'People would necessarily not feel comfortable with putting in their information unless they have probably had a familiarity with the website beforehand'.

If people use the site without logging in, then progress cannot be tracked and people will not be able to return to where they have left off during previous sessions. Furthermore, there are ethical dilemmas concerning interactive tasks where users provide personal information if the website is not password-protected [22].

One study focused on the privacy sector and its accessibility challenges [21]. Privacy policy terms and conditions can be difficult to understand for cognitively disabled persons. One participant who worked with people with dementia mentioned that they cannot give consent and that this group of people used substitute decision-makers. In this 
field, participants referred to some guidelines and privacy design, especially to the consent and confirmation process, as being more understandable [21]. In Johansson et al.'s [20] study, some participants described asking for help to understand web content or web logic as an invasion of privacy, e.g. paying invoices online.

\subsection{Content and language}

All of the studies revealed problems with content and language [20-26]. Many participants reported needing help to understand the content. Regarding more complex or specific information, especially related to privacy, people with disabilities often consult a lawyer or other needed support [21]. In Johansson et al.'s [20] study, some participants expressed a lack of confidence in their own abilities to understand and use resources on the web. It was a common way to ask for help from relatives or friends.

Singleton et al.'s [23] study was focused on how students with visual impairments navigate accessible Word and PDF documents. All of the participants in that study expressed frustrations regarding the provision of inaccessible instructional content by faculty members, especially image-only PDFs. These frustrations extended to both instructional content that was created by the faculty member (e.g., in Microsoft Word or PowerPoint) and content that was not developed by the faculty member (e.g., library database PDFs, supplemental applications or third-party websites).

There were a couple of studies related to the website's design, which attempted to clarify how people with different disabilities experienced the site design [20, 22]. In Watfern et al.'s [22] study, participants provided feedback about the accessibility of information on the website, particularly considering the different types of needs that users may have when engaging with textual and audio-visual information, e.g. if the website is not adaptable to a screen reader or the video content is not captioned. There were discussions about accessibility issues for culturally and linguistically diverse users. Using images and text needs more explanation. Both quick and easy access to certain content, and a linear, accumulative learning experience, could be contained within the same site [22].

One study was oriented to the learning environment where members with cognitive disabilities belong to the Code Club and were studying coding [24]. Some Code Club members had trouble reading and understanding the structure of code blocks and instructions about coding in the course material. There were colours in the code blocks, but while the colour-coding of blocks was generally helpful, members sometimes became reliant on them, which could limit their ability to understand the code itself [24].

González et al.'s [25] co-design process study outlined the type of support needs of people with intellectual disabilities. The study results showed that interactions should be focused and declared in an easy-read syntax or in plain language, depending on the context. Redundant elements can be detrimental to the construction of a precise meaning for the user. The pictograms must include contextual, characteristic and representative elements of the actions involved. Iconic and pictographic language (illustrations in general) is encouraged but must move away from children's language.

Crabb et al.'s [26] study was related to developers, students and their feelings about communication and content. When designing interfaces to deal with attention issues, participants commented that short precise information should be used and that easy-tounderstand instructions should be present. Breaking information into manageable chunks and using images could be used to assist in keeping the user attentive. Techniques that were used to assist in the area of communication accessibility, participants believed that 
techniques that were simple to accomplish were generally the ones that had the highest level of prevalence. Techniques such as subtitles, using recognizable icons, and simple typefaces are things that can be done to improve accessibility with a low level of difficulty [26].

\subsection{Designing digital services}

Four articles focused on digital service design accessibility [20, 23, 25, 26]. Participants in Johansson et al.'s [20] study found several elements that cause difficulties in using web pages. Most troublesome were pages with unwanted movements or flickering, a cluttered design, an evil design when the design is used to persuade or trick you into doing something, functions and services with a log-in, a lack of logic and the consequences of the concept and design and lack of trustworthiness [20]. Participants in Crabb et al.'s [26] study discussed elements that were not as prevalent. This included a large number of alterations in products to personalize the overall experience. Changes included alterations to colour, interface, language complexity, help offered and content organization.

Singleton et al.'s [23] study was focused on document accessibility, and the participants pointed out the importance of alt-text. One of the questions from the PDF observation referenced the alt-text embedded within an image of the document. The students with total blindness were able to answer this question correctly since screenreading software will read this information logically within the flow of the surrounding text. The students with low vision had difficulty with this question since these participants relied on tools (i.e. screen magnification or text-to-speech software or both) that do not read embedded alt-text. The students with low vision visually identified the bold or large font and surrounding white space, while the participants with total blindness listened for the screen reader to announce the heading elements. Participants in Crabb et al.'s [26] study mentioned methods such as larger text, larger buttons and having items in close proximity to each other for visually impaired people.

González at al.'s [25] study gathered the findings from participants into a heuristic guideline and summarized relevant elements that need to be taken into account when designing digital services. Interactions should anticipate their purpose transparently at the beginning, and the interactions must be sequential. Simple steps lead to the articulations of complex tasks. Designers need to avoid parallel actions or open situations that lead to ambiguity. Dialogues and interactions should be modulated in short meaningful steps, as they might be entry points for other dialogues within a modular architecture. Complex hierarchies of more than three levels should be avoided. Actions must be reversible and allow user mistakes and consider guided exploration. Dialogues and constructs should encourage user elicitation. In addition, navigation must always be consistent across the board.

Participants in Crabb et al.'s [26] study described items such as the implementation of text-to speech, auto-correcting and using suitable plain language as having moderate levels of difficulty and implementation prevalence. Techniques such as developing builtin colour overlays, neural interfaces and speech-to-text interpretation were all described as being difficult to implement and therefore had low levels of prevalence. Designers should use a consistent application layout, validation for any input and help guides as methods that could be used to assist users. 


\section{Discussions and limitations}

The purpose of this study was to review the qualitative literature on cognitive accessibility in a digital environment and areas of inquiry for future qualitative research in this context. The focus of this article was to identify qualitative research in the cognitive accessibility field. Even though the studies and articles were focused on people with disabilities, the same cognitive accessibility elements help everyone's digital service usage and provide a better customer experience. It would be beneficial to investigate the cognitive accessibility barriers for all people and not focus on only people who have disabilities. With the literature review results, it was possible to understand the barriers in the cognitive accessibility area, although the number of research studies was small.

All studies focused in some way on cognitive accessibility or its elements, such as proper language or user-centred design. That was the common element in all studies. An interesting observation was that the participant groups were different while most of the participant groups had some kind of disability. The articles and studies represented different challenges with cognitive accessibility elements. Where there were developers, teachers or people without any disabilities, the common factor was the need for accessibility education to be able to design accessible services. They were not sure which cognitive accessibility elements will benefit people with different disabilities [26].

It was interesting to note that there are not enough qualitative studies in this field. Cognitive accessibility as a subject is complex, and it is difficult to understand all the accessibility barriers without qualitative research where participants can explain the problems and barriers in using digital services. A co-design process with many methods could be helpful in understanding the challenges in this area. Now in this review, there were a couple of studies that used methods other than traditional interviews and observations.

An interesting observation was the problem with the log-in process, data privacy such as consent content and functionality. It requires more understandable design, content and logic. It is not a self-evident matter to make this process easier because this field is connected to strict data privacy as well as data protection regulations.

\subsection{Limitations and future research}

Although I used different databases for searching relevant articles, it is possible that some of the related articles do not appear in this review, for example, articles in different languages or with different wording. I only identified the articles with the exact search words, e.g. cognitive accessibility or accessibility. Future research should be more concentrated on overall cognitive accessibility-how all people, not just the disabled, will benefit from proper language and digital service design. Conducting more qualitative studies in the area of privacy and log-in processes to websites would be beneficial. It would be beneficial to use different search terms to identify cognitive accessibility elements such as language, navigation and user-centred design.

Digital services are changing continuously, and it is important to conduct more studies in the cognitive accessibility field. Considering the limited studies included in this review, it is recommended to conduct more studies and literature reviews. The result of this literature review may not represent the whole understanding of qualitative studies in the cognitive accessibility field. 


\section{Conclusion}

The result of this study was to identify the qualitative literature on cognitive accessibility in a digital environment and areas of inquiry for future qualitative research in this context. The literature review result was to also identify how common it was to mention cognitive accessibility in qualitative research articles.

The findings of this literature review led to the following conclusions:

1) Based on the results of the literature review, an understanding of existing research was obtained, as well as topics for further research in the cognitive accessibility field. There is a need for a deeper understanding by appreciating cognitive accessibility in the planning of digital services to help more effectively than the introduction and use of digital services.

2) There is a gap in qualitative researches in the cognitive accessibility field which are targeted to all people not only people with disabilities. Everybody appreciates easy-to-use services and understandable content. This viewpoint must be taken into consideration when planning qualitative accessibility research.

3) There is a need for further research in this field. Digitalization shapes our digital services and provides more digital platforms, applications and services. At the same time, we need to understand how to support these services by providing cognitive accessibility elements such as clear instructions and easy-to-use services.

According to the World Health Organization \& World Bank [27], around 15\% of the global population - over a billion people - live with some form of disability, and this number is expected to double to 2 billion by 2050. These people can require assistive technologies as well as accessible services. Counting the number of disabled people is not unambiguous. There is an implicit assumption that each 'type of disability' has specific health, educational, rehabilitation, social and support needs, but people with the same disability or impairment may have very different experiences and needs [27]. There is a growing demand for designing services that support all people.

\section{References}

[1] Case BJ. Universal Design. Harcourt policy report. 2003 [cited 2020 Dec 9]. Available from: http://images.pearsonclinical.com/images/PDF/assessmentReports/UniversalDesign_Rev1_Final.pdf

[2] Story MS. Maximizing Usability: The Principles of Universal Design. Assistive Technology, 1998; $10: 1,4-12$.

[3] Bertot J, Estevez E, Janowski T. Universal and Contextualized Public Services: Digital Public Service Innovation Framework. Government Information Quarterly 33, 2016; 2: 211-222.

[4] Seeman L, Cooper M, W3C board. Cognitive Accessibility User Research. Journal Title 66, 2016; 856890 .

[5] FAIDD. 2020 [cited 2020 Nov 11]. Available from: https://www.kehitysvammaliitto.fi/in-english/

[6] WebAim. Evaluating Cognitive Web Accessibility; 2020 [cited 2020 Dec 9]. Available from: http://webaim.org/articles/evaluatingcognitive 
[7] W3C. Web Content Accessibility Guidelines. 2018 [cited 2020 Dec 12]. Available from: https://www.w3.org/TR/WCAG21/\#wcag-2-1-supporting-documents

[8] Habil P, Trescher H. Accessibility for People with Cognitive Disabilities: An International Literature Review and Empirical Interview Study. 2018; 5. 60-68.

[9] Burmeister OK. Websites for seniors: Cognitive Accessibility. International Journal of Emerging Technologies and Society, 2010b; 8(2), 99-113

[10] Farrelly G. 'Practitioner Barriers to Diffusion and Implementation of Web Accessibility'. 1 Jan. 2011: 223-232.

[11] Lachal J, Revah-Levy A, Orri M, Moro MR. Metasynthesis: An Original Method to Synthesize Qualitative Literature in Psychiatry. 2017; Frontiers in Psychiatry. 8.

[12] Borg J, Lantz A, Gulliksen J. Accessibility to electronic communication for people with cognitive disabilities: a systematic search and review of empirical evidence. 2015. Univ Access Inf Soc 14, $547-$ 562

[13] Manzoor M, Vimarlund V. E-services for the social inclusion of people with disabilities: A literature review. Technology And Disability, 2017; 29(1-2), 15-33.

[14] Sintov N, Geislar S, White LV. Cognitive Accessibility as a New Factor in Proenvironmental Spillover: Results From a Field Study of Household Food Waste Management. Environment and Behavior, 2019; $51(1), 50-80$.

[15] Johansson; S, Gulliksen, J, Lantz, A. User Participation When Users have Mental and Cognitive Disabilities. In Proceedings of the 17th International ACM SIGACCESS Conference on Computers \& Accessibility (ASSETS '15). Association for Computing Machinery, New York, NY, USA, 2015; 6976.

[16] Keegan S. Qualitative research: Good decision making through understanding people, cultures and markets. London: Kogan Page; 2009. 11-15.

[17] Baumeister RF, Leary MR. 'Writing Narrative Literature Reviews', Review of General Psychology, 1997; 1(3), 311-320.

[18] Walsh; D, Downe, S. Meta-Synthesis Method for Qualitative Research: A Literature Review. Journal of Advanced Nursing 50: 2005; 2, 204-211.

[19] Zimmer L. Qualitative Meta-Synthesis: A Question of Dialoguing with Texts. Journal of Advanced Nursing 53, 2006; 3, 311-318.

[20] Johansson S, Gulliksen J, Lantz A. Cognitive Accessibility for Mentally Disabled Persons. 15th Human-Computer Interaction (INTERACT), Bamberg, Germany. 2015; 418-435.

[21] Cobigo V, Czechowski K, Chalghoumi H, Gauthier-Beaupre A, Assal H, Jutai J, Bah F. Protecting the privacy of technology users who have cognitive disabilities: Identifying areas for improvement and targets for change. Journal of Rehabilitation and Assistive Technologies Engineering 2020; 7.

[22] Watfern C, Heck C, Rule C, Baldwin P, Boydell KM. Feasibility and Acceptability of a Mental Health Website for Adults With an Intellectual Disability: Qualitative Evaluation JMIR Ment Health. 2019; $6(3)$

[23] Singleton KJ, Neuber KS. 'Examining How Students with Visual Impairments Navigate Accessible Documents', Journal of Visual Impairment \& Blindness, 2020; 114(5), 393-405.

[24] Koushik V, Kane SK. "It Broadens My Mind": Empowering People with Cognitive Disabilities through Computing Education. In Proceedings of the 2019 CHI Conference on Human Factors in Computing Systems (CHI '19). Association for Computing Machinery, New York, NY, USA, 2019; Paper 514, 112. 
[25] González HS, Córdova VV, Cid KE, Azagra MJ, Álvarez-Aguado I. Including intellectual disability in participatory design processes: Methodological adaptations and supports. In Proceedings of the 16th Participatory Design Conference 2020 - Participation(s) Otherwise - Volume 1 (PDC '20). Association for Computing Machinery: New York; 2020; 55-63.

[26] Crabb M, Heron M, Jones R, Armstrong M, Reid HY, Wilson A. Developing Accessible Services: Understanding Current Knowledge and Areas for Future Support. In Proceedings of the $2019 \mathrm{CHI}$ Conference on Human Factors in Computing Systems (CHI '19). Association for Computing Machinery, New York, NY, USA, 2019; Paper 216, 1-12.

[27] World Health Organization \& World Bank. World report on disability 2011. World Health Organization. 2011. [cited 2020 Dec 11]. Available from: https://apps.who.int/iris/handle/10665/44575 